\title{
The structure of graphs with Circular flow number 5 or more, and the complexity of their recognition problem
}

\author{
L. Esperet ${ }^{*} \quad$ G. Mazzuoccolo ${ }^{\dagger} \quad$ M. Tarsi ${ }^{\ddagger}$
}

September 10, 2015

\begin{abstract}
For some time the Petersen graph has been the only known Snark with circular flow number 5 (or more, as long as the assertion of Tutte's 5 -flow Conjecture is in doubt). Although infinitely many such snarks were presented eight years ago in [9], the variety of known methods to construct them and the structure of the obtained graphs were still rather limited. We start this article with an analysis of sets of flow values, which can be transferred through flow networks with the flow on each edge restricted to the open interval $(1,4)$ modulo 5 . All these sets are symmetric unions of open integer intervals in the ring $\mathbb{R} / 5 \mathbb{Z}$. We use the results to design an arsenal of methods for constructing snarks $S$ with circular flow number $\phi_{c}(S) \geq 5$. As one indication to the diversity and density of the obtained family of graphs, we show that it is sufficiently rich so that the corresponding recognition problem is NP-complete.
\end{abstract}

Keywords: Snarks, Circular flows, Nowhere-zero flows, NP-Completeness.

*Laboratoire G-SCOP (Grenoble-INP, CNRS), Grenoble, France. Partially supported by ANR Project Heredia (ANR-10-JCJC-0204-01), ANR Project Stint (ANR-13-BS020007), and LabEx PERSYVAL-Lab (ANR-11-LABX-0025).

†Università di Modena e Reggio Emilia, Dipartimento di Scienze Fisiche, Informatiche e Matematiche, Italy e-mail: mazzuoccolo@unimore.it

$\ddagger$ The Blavatnik School of Computer Science, Tel Aviv University, Israel e-mail: tarsi@post.tau.ac.il 


\section{Introduction}

For an integer $k$, a nowhere-zero $k$-flow in a graph $G$ is a flow in some orientation of $G$, such that the flow value on each arc is in $\{1,2, \ldots, k-1\}$. In this paper, we will be mainly interested in the following relaxation of nowhere-zero flows: for some real $r$, a circular nowhere-zero $r$-flow in a graph $G$ is a flow in some orientation of $G$, such that the flow value on each arc is in $[1, r-1]$. The circular flow number of a graph $G$ is the infimum of the reals $r$ such that $G$ has a circular nowhere-zero $r$-flow (more detailed definitions are presented in Section 3.1). It was conjectured by Tutte that any 2-edge-connected graph has a nowhere-zero 5-flow [13], and that any 4edge-connected graph has a nowhere-zero 3-flow [14]. A stronger conjecture of Jaeger [6] asserts that for any integer $k \geq 1$, any $4 k$-edge-connected graph has circular flow number at most $2+\frac{1}{k}$. These conjectures are wide open.

A particularly interesting class of graphs for studying the interplay between edge-connectivity and flows is the class of snarks, which are cyclically 4-edge-connected cubic graphs of girth at least 5, with no 3-edge-coloring (equivalently, with no circular nowhere-zero 4-flow). Because a snark is wellconnected, one might expect that it has circular flow number less than 5 . The Petersen graph shows that it is not the case, however it was conjectured by Mohar in 2003 that it is the only counterexample [10]. The conjecture was refuted in 2006 by Máčajová and Raspaud [9], who constructed an infinite family of snarks with circular flow number 5 .

The purpose of this paper is to show that the family $S_{\geq 5}$ of snarks with circular flow number at least 5 is significantly richer than just the set of graphs constructed by Máčajová and Raspaud. Observe that if a graph $G$ has circular flow number less than some value $k$, then $G$ admits a flow such that every arc has flow value in the open interval $(1, k-1)$. It is known to be equivalent to the fact that $G$ has a modulo $k$ flow such that in some orientation of $G$, every arc has flow value in $(1, k-1)$. With this observation in mind, we will make a systematic study of the set of modulo $k$ flow values that can be transferred through a two terminal network, where the flow on each edge is restricted to $(1, k-1)$. In Section 3 , we will show that for any two terminal network, this set of flow values is a symmetric union of open integer intervals in the ring $\mathbb{R} / k \mathbb{Z}$ (more will be said about these unions of intervals in Section 2). Moreover, the set $G I_{k}$ of unions of intervals that can be obtained in this way is closed under addition and intersection.

In Section 4 we will focus on the case $k=5$. Using ideas developed in 
the previous sections, we construct a certain set of two terminal networks allowing us to generate most of $G I_{5}$. In Section 5, these networks will be pieced together in several ways, allowing us to design a variety of methods for the construction of graphs (in particular snarks) of circular flow number at least 5. These constructions are not independent, so the same graphs can be obtained in several different ways (more will be said about the redundancy of our constructions in Section 5.3.1). Consequently, it is at first sight unclear how rich the constructed family is.

In Section 6, we will make this clear by showing that deciding whether a snark has circular flow number less than 5 is an NP-complete problem (and so the recognition problem for $S_{\geq 5}$ is co-NP-complete). We also generalize that result to any circular flow number $r, r \in(4,5]$. The proof uses the tools developed in Section 4. Many other nowhere zero flow related problems are known to be NP-hard: deciding the existence of nowhere zero 3-flow is NP-complete even if restricted to planar graphs (where it is equivalent to 3 -coloring of the dual graph); deciding the existence of nowhere zero 4-flow is NP-complete also when restricted to cubic graphs (where it is equivalent to 3-edge-coloring); when considering nowhere zero 5-flows, it was proved by Kochol [7] that if Tutte's 5-flow conjecture (mentioned above) is wrong, then deciding whether a cubic graph has a nowhere-zero 5-flow is an NP-complete problem. It was also proved in [3] that for any $t$ and $k$, either all $t$-edgeconnected planar (multi) graphs have circular flow number at most $2+\frac{1}{k}$, or deciding whether a $t$-edge-connected planar (multi) graph has circular flow number at most $2+\frac{1}{k}$ is an NP-complete problem (this is related to the conjecture of Jaeger mentioned above).

Our result, however, is significantly different. Only a limited family of snarks with flow number 5 (or more) where so far known and here we show that the family of such graphs is rich enough to yield NP-hardness. Our result does not rely on any unsettled conjecture. If Tutte's conjecture indeed holds, then this is the family of snarks whose flow number is precisely 5 . Another unique aspect of our proof is the need to restrict the construction to actually provide snarks, unlike other proofs which strongly rely on graphs with smaller girth and connectivity.

In Section 7, we conclude with some open problems and suggestions for further research. 


\section{The algebra of symmetric unions of open integer intervals in $\mathbb{R} / k \mathbb{Z}$}

Definition 1. Let $r$ be a positive real number. $\mathbb{R} / r \mathbb{Z}$ denotes the ring of real numbers modulo $r . \mathbb{R} / r \mathbb{Z}$ is commonly represented by a cycle of length $r$ where the numbers of the real interval $[0, r)$ are cyclically ordered clockwise from 0 to $r=0$. An interval $(a, b)$ on $\mathbb{R} / r \mathbb{Z}$ refers to the set of numbers covered when traversing clockwise from a to $b$. Closed and half closed intervals are similarly defined. It follows, for example, that $0 \in(a, b)$ if and only if, when referred to as real numbers, $a>b$. Also the union of the disjoint intervals $[a, b)$ and $[b, a)$ is always the entire cycle $\mathbb{R} / r \mathbb{Z}$. Traversing clockwise from $a$ to $b$ is ambiguous when $a=b$. We resolve this by taking the long way, so $(a, a)=\mathbb{R} / r \mathbb{Z}-\{a\}$ (and not the empty set).

The following relates to $\mathbb{R} / k \mathbb{Z}$ where $k$ is a positive integer.

Definition 2. An integer open interval of $\mathbb{R} / k \mathbb{Z}$ is any interval $(a, b)$ where $a$ and $b$ are (not necessarily distinct) integers. There are clearly $k^{2}$ such intervals with $a, b \in\{0,1, \ldots, k-1\}$. The set of all integer intervals of $\mathbb{R} / k \mathbb{Z}$ is denoted here by $I_{k}$.

Definition 3. A set $A \in \mathbb{R} / k \mathbb{Z}$ is symmetric if and only if $a \in A \Leftrightarrow-a(=k-a) \in A$.

Definition 4. Let $S I_{k}$ denote the set of all unions of subsets of $I_{k}$ which form symmetric subsets of $\mathbb{R} / k \mathbb{Z}$.

For example, 0 and 1 are the only integers in $\mathbb{R} / 2 \mathbb{Z}$ and accordingly, $I_{2}=\{(0,0),(0,1),(1,0),(1,1)\}$ and

$$
S I_{2}=\{\emptyset,(0,1) \cup(1,0),(0,0),(1,1), \mathbb{R} / 2 \mathbb{Z}\}
$$

All four non-empty sets in $S I_{2}$ contain every non-integer and they only differ by a different subset of $\{0,1\}$ that each of them contains. Starting with $\left|S I_{1}\right|=3$ and $\left|S I_{2}\right|=5$, it is not hard to verify that $\left|S I_{k}\right|$ are Fibonacci numbers. Coming next are $\left|S I_{3}\right|=8,\left|S I_{4}\right|=13$ and $\left|S I_{5}\right|=21$.

Proposition 5. $S I_{k}$ is clearly closed under the following set operations: 
- Addition, defined by $A+B=\{a+b \mid a \in A$ and $b \in B\}$. As the involved sets are symmetric, addition can be replaced by subtraction (yet $A+A=A-A$ is neither "0", nor empty and addition is not invertible);

- Set Intersection, $A \cap B$;

- Set Union, $A \cup B$;

- Open complement. The open complement of an open set $A$ is the complement of its closure $\sigma(A)$, that is, $\overline{\sigma(A)}=\mathbb{R} / k \mathbb{Z}-\sigma(A)$. Following that definition, $A \cup \overline{\sigma(A)}$ is generally not the entire cycle, but lacks some integers.

In what follows addition and intersection play the major role.

\section{Applications to the theory of circular nowhere- zero flows}

\subsection{Circular nowhere-zero flows}

Definition 6. Given a real number $r \geq 2$, a circular nowhere-zero $r$ flow ( $r$-CNZF for short) in a graph $G=(V, E)$ is an assignment $f: E \rightarrow$ $[1, r-1]$ and an orientation $D$ of $G$, such that $f$ is a flow in $D$. That is, for every vertex $x \in V, \sum_{e \in E^{+}(x)} f(e)=\sum_{e \in E^{-}(x)} f(e)$ where $E^{+}(x)$, respectively $E^{-}(x)$, are the sets of edges directed from, respectively toward, $x$ in $D$.

Accordingly defined is:

Definition 7. The circular flow number $\phi_{c}(G)$ of a graph $G$ is the infimum of the set of numbers $r$ for which $G$ admits an $r$-CNZF. If $G$ has a bridge then we define $\phi_{c}(G)=\infty$.

The notion of $r$-CNZF was first introduced in [4], observing that $(k, d)$ coloring, previously studied by Bondy and Hell [1], can be interpreted as the dual of real (rather than integer)-valued nowhere-zero flow. Integer nowhere-zero flows are much more widely known and intensively studied since first presented by W. Tutte [13] 60 years ago. A comprehensive source for material on integer flows and related topics is C.Q. Zhang's book [15]. 
A circular nowhere-zero modular- $r$-flow $(r-\mathrm{MCNZF})$ is an analogue of an $r$-CNZF, where the additive group of real numbers is replaced by the additive group of $\mathbb{R} / r \mathbb{Z}$.

Definition 8. An $r$-MCNZF in a graph $G=(V, E)$ is an assignment $f$ : $E \rightarrow[1, r-1] \subseteq \mathbb{R} / r \mathbb{Z}$ and an orientation $D$ of $G$, such that for every vertex $x \in V, \sum_{e \in E^{+}(x)} f(e)=\sum_{e \in E^{-}(x)} f(e)$. Summation is performed in $\mathbb{R} / r \mathbb{Z}$.

Part of the definition of an $r$-CNZF is an orientation where all flow values are positive. Since there are no "positive" or "negative" numbers in $\mathbb{R} / r \mathbb{Z}$, the orientation where an $r$-MCNZF $f$ is defined is only required "for reference". The direction of an edge $e$ can be reversed and $f$ transformed into another $r$-MCNZF, where $f(e) \in \mathbb{R} / r \mathbb{Z}$ is replaced by $-f(e) \in \mathbb{R} / r \mathbb{Z}$. As a measure to relate to different orientations we define:

Definition 9. Let $f$ be an $r-\mathrm{MCNZF}$ in a reference orientation $D_{0}$ of a graph $G$. We refer by $f_{D}$ to the flow, on an orientation $D$, defined by $f_{D}(e)=f(e)$ for edges e of the same orientation in $D_{0}$ and $D$, and $f_{D}(e)=-f(e)$ if the directions of $e$ in $D_{0}$ and $D$ differ.

The following result can be easily deduced from Tutte's original work on integer flows [13] and it is also explicitly stated in some more recent literature, e.g. [11]

Proposition 10. The existence of a circular nowhere-zero $r$-flow in a graph $G$ is equivalent to that of an $r$-MCNZF.

Furthermore, if $f$ is an $r$-MCNZF in (an orientation $D_{0}$ ) of $G$, then there exists an orientation $D$ of $G$ and a (real valued positive) $r$-CNZF $g$ in $D$, such that for every edge e of $G, g(e) \equiv f_{D}(e)$ modulo $r$.

Consequently,

Proposition 11. For any graph $G=(V, E), \phi_{c}(G)<r$ if and only if there exists an $r$-MCNZF $f$ in $G$ such that $f: E \rightarrow(1, r-1)$. Accordingly, we refer to such a flow $f$ as a sub- $r$-MCNZF.

Proof. If $\phi_{c}(G)=r-\epsilon<r$, then there exists a flow $g$ in $G, g: E \rightarrow$ $[1, r-1-\epsilon]$. For a small enough $\delta,(1+\delta) g: E \rightarrow(1, r-1)$. When its values are interpreted as modulo $r$ residues, $(1+\delta) g$ yields an $r$-MCNZF with range $(1, r-1)$ modulo $r$, as claimed. On the other hand, if $f: E \rightarrow(1, r-1)$ is a sub- $r$-MCNZF, then, by Proposition 10 , there exists an $r$-CNZF $g$ such that 
$g: E \rightarrow(1, r-1) \subseteq \mathbb{R}$. Recall that if $t \in(1, r-1)$ modulo $r$, then both $t$ and $r-t$ belong to $(1, r-1)$ as real numbers. Then $g$ is clearly an $r^{\prime}$-CNZF for some $r^{\prime}<r$, so $\phi_{c}(G)<r$.

\subsection{Generalized edges}

Definition 12. A generalized edge (g-edge for short) $G_{u, v}$ is simply a graph $G=(V, E)$ and two of its vertices $u$ and $v$. The vertices $u$ and $v$ are the terminals of $G_{u, v}$. Using g-edges, as well as more generalized similar structures is a rather common technique, when dealing with circular NZF's, e.g [11, 9, 8, 5] where g-edges are called "dipoles", "two terminal networks" and "2-poles".

Our interest in g-edges results from the following parameter:

Definition 13. The open $r$-capacity $C P_{r}(q)$ of a $g$-edge $q=G_{u, v}$ is a subset of $\mathbb{R} / r \mathbb{Z}$, defined as follows: Add to $G$ an additional edge $e_{0} \notin E(G)$ with endvertices $u$ and $v$, and set:

$C P_{r}\left(G_{u, v}\right)=\left\{f\left(e_{0}\right) \mid f\right.$ is a modulo $r$ flow in $G \cup e_{o}$ and $\left.f: E(G) \rightarrow(1, r-1)\right\}$

More visually, $C P_{r}\left(G_{u, v}\right)$ is the set of all flow values (in $\mathbb{R} / r \mathbb{Z}$ ) which can be "pushed" through $G$ from source $u$ to sink $v$, under all orientations of $G$, where the "flow capacity" of every edge of $G$ is restricted to $(1, r-1)$.

A seemingly more natural parameter is the closed $r$-capacity, where the set of allowed flow values is the entire closed interval [1, $r-1]$. The notion of a closed capacity is derived from the definition of an $r$-MCNZF. It is extensively used in [11] to construct graphs $G$ with $\phi_{c}(G)=r$, for any rational $4<r<5$. Despite the similar definitions, open capacities are not straightforwardly obtained from closed ones. If the closed capacity of a gedge contains a closed interval $[a, b]$, the open capacity of the same g-edge does not necessarily contain the open interval $(a, b)$, and it might even be disjoint from $[a, b]$. Closed capacities do not serve our needs, as we focus on sub- $r$-MCNZF's, in particular sub-5-MCNZF. No g-edge is known of closed 5 -capacity other than $(1,4)$, or $\mathbb{R} / 5 \mathbb{Z}$. Considering the 5 -flow conjecture, it might well be the case that none exists. In what follows we only deal with open capacities and omit the term "open" when referring to one.

The notion of g-edge relates to $S I_{k}$ via the following basic property of $k$-flows: 
Lemma 14. $C P_{k}\left(G_{u, v}\right) \in S I_{k}$, for any integer $k \geq 2$ and a g-edge $G_{u, v}$.

Proof. Take any $t \in C P_{k}\left(G_{u, v}\right)$. By definition, there exists a modulo $k$ flow $f$ in an orientation of $H=G \cup u v$, such that $f: E(G) \rightarrow(1, k-1)$ and $f(u v)=t$. For a given $\epsilon>0$ we select $0<\delta<\epsilon /|E(G)|$. We also make sure that $\delta$ is small enough, so that $f: E(G) \rightarrow(1+\delta, k-1-\delta)$. It follows that $f: E(G) \rightarrow[1+\delta, k-1-\delta]$, and, for that closed interval, no edge $e \in E(G)$ is saturated by $f$, in the sense that $f(e)$ reaches neither of the bounds $1+\delta$ and $k-1-\delta$. We now keep the orientation unchanged and apply a "Max flow" algorithm on the network $G$ with source $u$ and sink $v$, starting from $f$, in both directions. Although arithmetic is modulo $k$, the relevant elements of Network flow theory (with upper and lower capacities) are still valid: Increasing, respectively decreasing, translates to moving clockwise, respectively counterclockwise. An augmenting path is a path from $v$ to $u$, where the current flow on an edge can be increased by pushing it toward $k-1-\delta$ if the edge has the direction of the path, or toward $1+\delta$ if the edge is of the opposite direction. Starting with the flow $f$, the flow on $u v$ can be continuously augmented (say, by repeatedly selecting a shortest augmenting path, to guarantee termination), until a saturated edge-cut is reached. Similarly, $f$ can be continuously decreased along decreasing paths (from $u$ to $v$, where the roles of the bounds $k-1-\delta$ and $1+\delta$ are switched), until a cut is saturated in the opposite direction. In a saturated cut obtained by augmenting $f(u v)$, the flow values are $k-1-\delta$ on $m_{1}$ edges, directed forward, and $1+\delta$ on $m_{2}$ edges, directed backwards. The "max" (modulo $k$ ) flow on $u v$ at that stage is $m_{1}(k-1-\delta)-m_{2}(1+\delta)=-\left(m_{1}+m_{2}\right)-$ $\left(m_{1}+m_{2}\right) \delta$. Clearly $a=-\left(m_{1}+m_{2}\right)$ is an integer and $\left(m_{1}+m_{2}\right) \leq|E(G)|$, so $\left(m_{1}+m_{2}\right) \delta<\epsilon$. In this manner, the flow $t$ on $u v$ can be continuously increased to $a-\epsilon$ where $a$ is an integer, and $\epsilon$ any small positive number. Similarly, it can be continuously decreased from $t$ to $b+\epsilon$ for an integer $b$. In summary, for every small $\epsilon$, there exist integers $a$ and $b$, such that $t \in(b+\epsilon, a-\epsilon) \subseteq C P_{k}\left(G_{u, v}\right)$. This implies that, if $t$ is not an integer, then the entire open unit interval that includes $t$ is contained in $C P_{k}\left(G_{u, v}\right)$. If $t$ is an integer then both open unit intervals on its two sides are contained in $C P_{k}\left(G_{u, v}\right)$. It follows that $C P_{k}\left(G_{u, v}\right)$ is indeed a union of open integer intervals. Note that the value $-t$ is obtained by the flow $-f$, so $C P_{k}\left(G_{u, v}\right)$ is also symmetric.

In what follows we allow the edge set of a graph to consist of both (simple) edges and g-edges, by means of the following convention: A g-edge $q=H_{u, v}$ 
in a graph $G$ is a subgraph $H$ of $G$ that shares its terminals $u$ and $v$ with the rest of the graph, and is otherwise vertex-disjoint from $G-H$. If $f$ is a flow in $G$, then $f(q)$ denotes the amount of flow that "traverses from $u$ to $v$ (or the other way around) through the subgraph $H$ ". When considering sub- $k$ MCNZF's, the main characteristic of a g-edge is its $k$-capacity. With that in mind, we refer to a g-edge $q$ with terminals $u$ and $v$ and $k$-capacity $A \in S I_{k}$ as an $A$-edge, $q=u v$ without elaborating any further on its structure. For that matter, a $(1, k-1)$-edge, may, or may not be a simple edge. Any other capacity implies a genuine generalized edge. A flow $f$ is a sub- $k$-MCNZF if and only if for every edge $e$, simple or generalized, $f(e) \in C P_{k}(e)$.

Definition 15. We say that a set $A \in S I_{k}$ is graphic, if there exists a g-edge with $k$-capacity $A$. The set of all graphic members of $S I_{k}$ is denoted here by $G I_{k}$.

Rather obvious, yet fundamental observations are:

\section{Proposition 16.}

- Let $q$ with $k$-capacity $A$ and $t$ with $k$-capacity $B$ be two g-edges, sharing a pair of terminals $u$ and $v$ and otherwise disjoint. The union of $q$ and $t$ is called the parallel join of $q$ and $t$ and it forms a new g-edge with terminals $u$ and $v$ and $k$-capacity $A+B$ (see Proposition 5 for the definition of $A+B$ ).

- The union of two g-edges $q=u v$ with $k$-capacity $A$, and $t=v w$ with $k$-capacity $B$, which share a single terminal $v$ forms the serial join of $q$ and $t$. That is a new g-edge with terminals $u$ and $w$ and $k$-capacity $A \cap B$.

- It follows that the subset $G I_{k}$ of all graphic members of $S I_{k}$ is closed under Addition and Intersection and as such, it is a sub-algebra of $S I_{k}$ with respect to these two operations.

Let us demonstrate the above by an analysis of the algebra $G I_{3}$. The $k$ capacity of a simple edge is, by definition, $(1, k-1)$. For $k=3$, that is $(1,2)$. The $(+, \cap)$-algebra generated by $(1,2)$ includes the following 6 members:

- $(1,2)$

- $(2,1)=(1,2)+(1,2)$ 
- $(0,0)=(1,2)+(2,1)$

- $\mathbb{R} / 3 \mathbb{Z}=(0,0)+(1,2)$

- $\emptyset=(1,2) \cap(2,1)$

- $(2,0) \cup(0,1)=(0,0) \cap(2,1)$

We tend to believe that the remaining two sets in $S I_{3}$, namely $\mathbb{R} / 3 \mathbb{Z}$ $\{1,2\}$ and $\mathbb{R} / 3 \mathbb{Z}-\{0,1,2\}$ are not graphic, yet, at this point, we have no serious evidence to support such a claim.

\section{$4 \quad G I_{5}$ and some related observations}

Definition 17. Associated with a set $A \in S I_{k}$ are two size parameters: its amplitude $A m(A)$, which is the length (number of unit intervals) of the smallest interval that contains $A$, and its measure $\operatorname{Me}(A)$, which is the number of unit intervals contained in $A$.

In this section, the capacity of a g-edge refers to its open 5-capacity

\subsection{Generating $G I_{5}$}

Included in $G I_{5}$ are

- $(1,4)$ of amplitude and measure 3 , represented by a simple edge. We will later build additional $(1,4)$-edges, to serve some needs (related mostly to edge-connectivity), of specific constructions.

- $\mathbb{R} / 5 \mathbb{Z}=(1,4)+(1,4)$ of amplitude and measure 5

These two sets form a closed sub-algebra, so another generator is required in order to go further. Such a generator is the 5-capacity of $\mathcal{P}_{10}^{*}(u, v)$, the graph obtained from the Petersen graph $\mathcal{P}_{10}$ by removing an edge $u v$ (Any other graph $G$ with $\phi_{C}(G)=5$, that reduces to less than 5 when an edge is removed, can be used instead of $\left.\mathcal{P}_{10}\right)$. Since $\phi_{c}\left(\mathcal{P}_{10}\right)=5$, the capacity of $\mathcal{P}_{10}^{*}(u, v)$ is disjoint from $(1,4)$ and therefore a subset of $(4,1)$. On the other hand, $\phi_{c}\left(\mathcal{P}_{10}^{*}\right)<5$, which implies that 0 is included in the capacity. The only set in $S I_{5}$ which meets these two conditions is $(4,1)$. More sets of $G I_{5}$ can now be generated: 
- $(4,1)$ of amplitude and measure 2.

- $\emptyset=(1,4) \cap(4,1)$ of amplitude and measure 0

- $(3,2)=(4,1)+(4,1)$ of amplitude and measure 4

- $(0,0)=(4,1)+(1,4)$ of amplitude and measure 5

- $(4,0) \cup(0,1)=(4,1) \cap(0,0)$ of amplitude and measure 2

- $(3,0) \cup(0,2)=(0,0) \cap(3,2)$ of amplitude and measure 4

- $(1,2) \cup(3,4)=(3,2) \cap(1,4)$ of amplitude 3 and measure 2

- $\mathbb{R} / 5 \mathbb{Z}-\{1,4\}=((1,2) \cup(3,4))+((1,2) \cup(3,4))$ of amplitude and measure 5

- $\mathbb{R} / 5 \mathbb{Z}-\{0,1,4\}=(\mathbb{R} / 5 \mathbb{Z}-\{1,4\}) \cap(0,0)$ of amplitude and measure 5

- $(3,2)-\{1,4\}=(3,2) \cap(\mathbb{R} / 5 \mathbb{Z}-\{1,4\})$ of amplitude and measure 4

- $(3,2)-\{0,1,4\}=(3,2) \cap(\mathbb{R} / 5 \mathbb{Z}-\{0,1,4\})$ of amplitude and measure 4

Once again, the serial-parallel routine ceases to produce new results and an additional tool is required.

Consider a vertex $x$ of degree 3 where one of the three edges incident with $x$ is of capacity $B \subseteq(1,4)$. Let the orientation of the other two edges $a$ and $b$ be such that one of them is outgoing from $x$ and the other one is ingoing. Let $f$ be a sub-5-MCNZF. For the difference between $f(a)$ and $f(b)$ to lie in $B \subseteq(1,4)$, these two values cannot belong to the same unit interval. As a consequence:

Lemma 18. Let $P$ be a path with at least one internal vertex in a graph $G$, such that: all edges of $P$ are of the same capacity $A$, of measure $M e(A)=2$; every internal vertex $v$ has degree three and the third edge incident to $v$ (the one not in $P$ ) is of some capacity $B_{v} \subseteq(1,4)$ (in particular a simple edge). Assume an orientation of $G$ where $P$ is a directed path. If $f$ is a sub-5MCNZF in $G$, then the values of $f$ along $P$ are alternating between the two unit intervals contained in $A$.

Alternating values along an odd cycle bear a contradiction, which implies the following two conclusions: 
Corollary 19. Let $C$ be an odd cycle in a graph $G$, along vertices of degree 3. If all edges of $C$ are of the same capacity $A$, of measure $\operatorname{Me}(A)=2$, and the third edges incident with each vertex of $C$ is of some (not necessarily the same) capacity $B \subseteq(1,4)$ (in particular a simple edge), then $\phi_{c}(G) \geq 5$.

and

Corollary 20. Let $C$ be an odd cycle in a graph $G$, along vertices of degree 3 , such that: all edges of $C$ are of the same capacity $A$, of measure $M e(A)=2$, and the third edge incident with each vertex of $C$ is of capacity $B \subseteq(1,4)$ (in particular a simple edge). The deletion of an edge uv of $C$ results in a g-edge $q=G_{u, v}$ such that $C P_{5}(q) \subseteq \overline{\sigma(A)}$.

Corollary 20 now allows us to construct a 3-edge-connected (1,4)-edge, to be later used as a replacement for single edges, when higher connectivity is required:

Definition 21. A thick (1,4)-edge with terminals $u$ and $v$ is obtained from a copy of $K_{4}$ where two edges of a triangle are replaced by $(4,1)$-edges, and the third edge, uv, of that triangle is removed.

By Corollary 20, the capacity of the obtained g-edge with terminals $u$ and $v$ (see Figure 1, top right) is a subset of $\overline{\sigma(4,1)}=(1,4)$. It is easy to verify that 2 belongs to the obtained capacity, which is therefore, indeed $(1,4)$ (no set in $S I_{5}$ which is a proper subset of $(1,4)$ includes the point 2$)$.

Following the exact same lines with the capacity $(4,1)$ replaced by $(1,2) \cup$ $(3,4)$, Corollary 20 can be used to further broaden the list of sets in $G I_{5}$. If two edges of a triangle of $K_{4}$ are replaced by $(1,2) \cup(3,4)$-edges, and the third edge $u v$ of that triangle is removed, then, by Corollary 20, the capacity $D$ of the obtained g-edge with terminals $u$ and $v$ (see Figure 1) is a subset of $\overline{\sigma((1,2) \cup(3,4))}=(4,1) \cup(2,3)$. It is easy to verify $0 \in D$ and $\frac{5}{2} \in D$, so $D=(4,1) \cup(2,3)$.

New members can now be added to $G I_{5}$

- $(4,1) \cup(2,3)$, of amplitude 4 and measure 3

- $(4,0) \cup(0,1) \cup(2,3)=((4,1) \cup(2,3)) \cap(0,0)$, of same amplitude and measure

- $(2,3)=((4,1) \cup(2,3)) \cap(1,4)$, of amplitude and measure 1 
We have listed, so far, 16 members of $G I_{5}$. The remaining 5 sets in $S I_{5}$ are obtained by removing $\{2,3\}$ from the 5 sets in our list that contain $\{2,3\}$ as a subset. A similar phenomenon was observed in $G I_{3}$. We tend to believe these 5 sets are not graphic, but so far, we have nothing to support that claim.

$(1,4)$

$(4,1)$

$(3,2)$

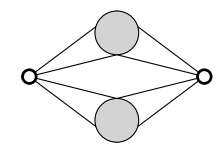

$(0,0)$
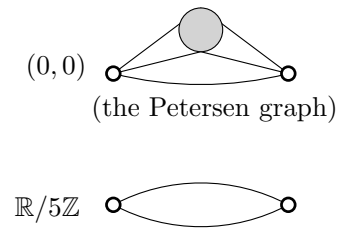
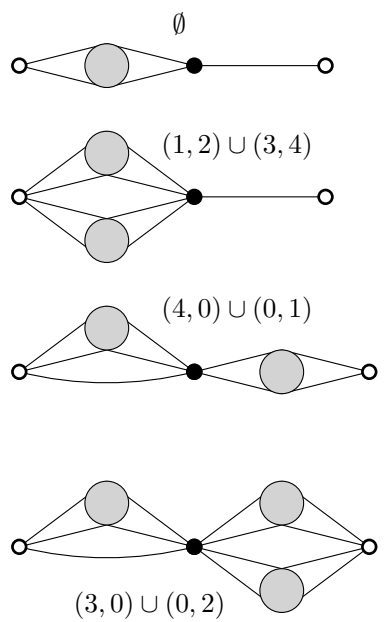
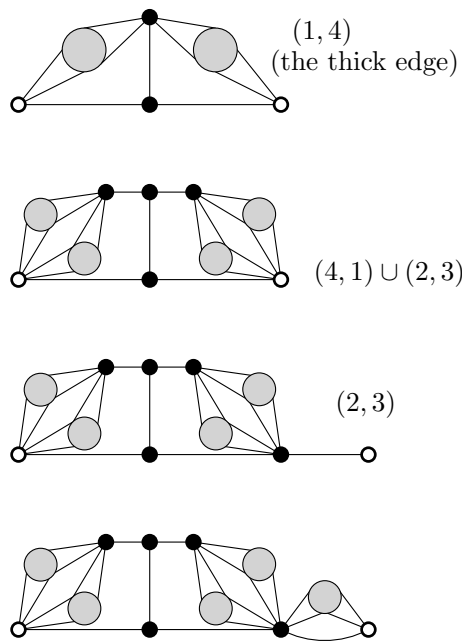

$(4,0) \cup(0,1) \cup(2,3)$

Figure 1: Basic generalized edges

Figure 1 shows g-edges for twelve capacities (two for $(1,4)$ ) out of the sixteen on our list. Next to the diagram of each is its 5-capacity. Terminal vertices are depicted by white dots. The repeatedly used $\propto 0<$ shaped pattern stands for the $(4,1)$-edge $\mathcal{P}_{10}^{*}(u, v)$ obtained from $\mathcal{P}_{10}$ by the removal of an edge $u v$.

We conclude our arsenal of $G I_{5}$ related observations with the following schema:

Lemma 22. Let $C$ be a cycle, consisting of simple edges, in a graph $G$. Let $T \subseteq G I_{5}$ be a set of 5-capacities, such that the amplitude of their union is at most 3. Let $G^{\prime}$ be obtained from $G$ by replacing every edge of $C$ by an $A$-edge, for some (not necessarily the same) $A \in T$. If $\phi_{c}(G) \geq 5$, then also $\phi_{c}\left(G^{\prime}\right) \geq 5$.

Proof. Assume, to the contrary that there exists a sub-5-MCNZF $f$ in $G^{\prime}$. Since the union of all members of $T$ is of amplitude at most 3 , there exists 
$t \in \mathbb{R} / 5 \mathbb{Z}$ such that for every $y \in \bigcup_{A \in T} A, y+t \in(1,4)$. Let $f_{1}$ be a flow in $G^{\prime}$, defined by $f_{1}(e)=t$ if $e$ belongs to $C$, and $f_{1}(e)=0$ for all other edges $e$. The flow value of $f+f_{1}$ belongs to $(1,4)$ for every edge of $C$ and $f+f_{1}$ is identical to $f$ on the other edges. $f+f_{1}$ is therefore, a sub-5-MCNZF in the original graph $G$, which is a contradiction if $\phi_{c}(G) \geq 5$.

\section{Explicit construction of graphs $G$, in par- ticular snarks, with $\phi_{c}(G) \geq 5$}

Let $F_{\geq 5}$ stand for the set of graphs $G$ with $\phi_{c}(G) \geq 5$, and let $S_{\geq 5}$ be the set of all snarks in $F_{\geq 5}$. Recall that a snark is a 3-regular graph $S$, cyclically 4-edge-connected, of girth 5 or more and circular flow number $\phi_{c}(S)>4$.

For some time, $S_{\geq 5}$ was conjectured to consist solely of the Petersen graph [10, until an infinite family of such snarks was presented in [9]. Similar constructions, aimed toward different goals, can also be found in other articles, e.g [5]. Nonetheless, we now demonstrate that $S_{\geq 5}$ is in fact much richer than that. The concepts and tools, developed on the previous section, give rise to a large variety of snarks in $S_{\geq 5}$. Let us first note that a graph $G \in F_{\geq 5}$, of the right girth and connectivity, can be transformed into a snark in $S_{\geq 5}$, by means of:

Definition 23. Given a graph $G$, an expansion of a vertex $x$ into a graph $X$ is obtained by: Deleting the vertex $x$ from $G$ and replacing it by the graph $X$. Each edge yx of $G$ is replaced by an edge between $y$ and an arbitrary vertex of $X$.

Proposition 24. Let $G^{\prime}$ be obtained by a vertex expansion of a graph $G=$ $(V, E)$ and let $f$ be a flow in $G^{\prime}$, then the restriction of $f$ to $E$ is a flow in $G$. Consequently, $\phi_{c}\left(G^{\prime}\right) \geq \phi_{c}(G)$. In particular, if $G \in F_{\geq 5}$, so is $G^{\prime}$.

Expansion can be accompanied by:

Definition 25. Smoothing a vertex $x$ of degree $d(x)=2$ means the removal of $x$, while merging the two edges ux and $x v$ incident with $x$, into a single new edge uv.

Observe that smoothing a vertex does not affect flow values.

Considering the above, we focus on constructing cyclically 4-edge-connected graphs in $F_{\geq 5}$, of girth at least 5. Each such graph can then be transformed 
into infinitely many snarks $S \in S_{\geq 5}$, by selecting proper expansion graphs (almost) arbitrarily. An expansion graph $X$ should not necessarily be highly connected. Edges by which $X$ is attached to the rest of the graph can be tailored to repair small edge-cuts. The graph $X$ is not even required to be connected (see Figure 2). Let us now briefly describe some actual constructions, based on these principles :

\subsection{Constructions based on Corollary 19}

Corollary 19 lets us turn any arbitrarily selected non-bipartite cubic graph $G$, which is "almost" cyclically 4-edge-connected, of girth "almost" $\geq 5$ into a snark, by replacing the edges of any odd cycle $C$ by $A$-edges, of measure $\operatorname{Me}(A)=2$, and then properly expanding vertices of degree $>3$. We used the term "almost", because the length of $C$ can be less than 5 , yet larger than that, when simple edges are replaced by g-edges. The same holds for small edge-cuts, which includes edges of $C$. There are three different sets of measure 2 in $G I_{5}$. The initial graph $G$ and expansion graphs for vertices of large degrees can be arbitrarily selected. The family of obtained members of $S_{\geq 5}$, by means of this method only is already rather diverse and rich.

Here are some of the smallest possible examples: Replace the three edges of a triangle in $K_{4}$, by $(4,1)$-edges, $\mathcal{P}_{10}^{*}(u, v)$, to obtain the graph drawn in Figure 2, left. By Corollary 19 the obtained graph belongs to $F_{\geq 5}$. It includes 3 vertices of degree 5 , which should be expanded in order to obtain a snark.
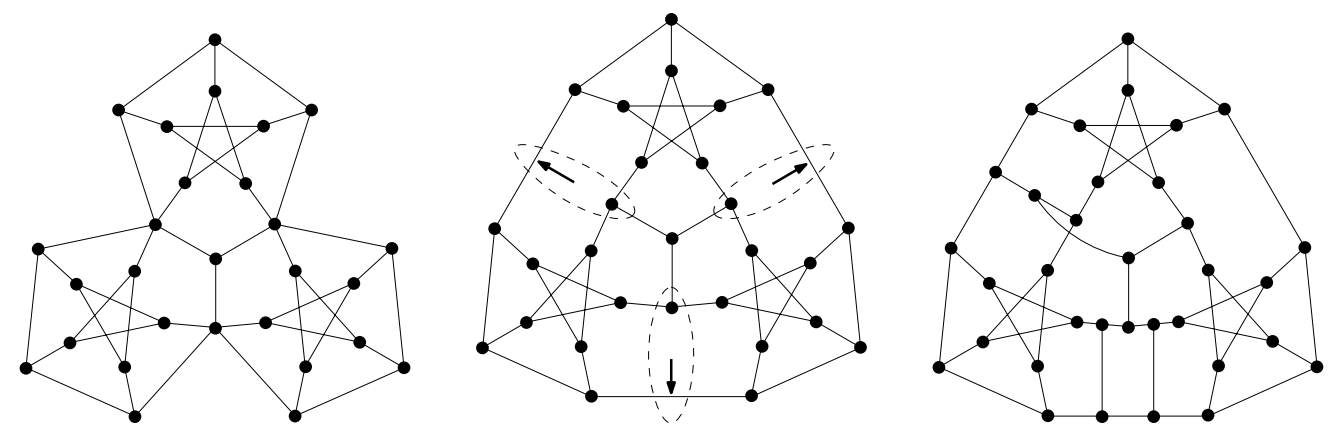

Figure 2: $K_{4}$ with a triangle consisting of $(4,1)$-edges (left); $S_{28}$ (center); and three distinct expansion graphs applied to the same source graph (right).

The snark in Figure 2 (center) (Let's call it $S_{28}$, not an official name) is obtained by expanding each of the 3 vertices of degree 5 of the graph in 
Figure 2 (left) into a graph which consists of two isolated vertices. Each arrow in the diagram points at a location of a missing second vertex, which was removed by smoothing. The graph $S_{28}$ was identified as a snark during a computerized survey [2], to discover all snarks of order 30 or less. The circular flow number of the graph was calculated by Máčajová, and Raspaud 9] - also by means of a computer program. They identified $S_{28}$ as the smallest snark in $S_{\geq 5}$, other than $\mathcal{P}_{10}$. The graph does not comply with the construction method developed in 9 .

Two more snarks in $S_{\geq 5}$ where discovered by means of a computer, both of order 30. These are obtained by replacing one of the expansion graphs in $S_{28}$ by a path $P_{3}$. There are indeed two non-isomorphic patterns to do so with cyclically 4-edge-connectivity retained. $P_{3}$ is one of the expansion graphs of the snark on Figure 2 (right).

\subsection{Constructions based on Lemma 22}

This method seems similar to the one obtained from Corollary 19, That similarity is somewhat misleading. Here the initial graph $G$ is not arbitrary. The selection of $G$ is restricted to previously constructed members of $F_{\geq 5}$. On the other hand, this method is not limited to odd cycles and the set $T$ of replacement capacities is richer. $T$ can either contain a single capacity of amplitude 3 or less, and there are six distinct such capacities in our list of members of $G I_{5}$, or it can consist of more than one set, such as $T=\{(4,0) \cup(0,1),(4,1)\}, T=\{(1,2) \cup(3,4),(2,3),(1,4)\}$ and many more. Needless to say, expansion graphs can be freely chosen while applying this method, just as well. Lemma 22, because it is weaker than Corollary 19, need to be recursively applied to previously constructed graphs $G \in S_{\geq 5}$. Such a recursion would be redundant for Corollary 19, where any initial graph $G$ can be selected to start with. Yet, new members of $S_{\geq 5}$ can replace the Petersen graph in producing distinct g-edges for the same capacities, which opens many routes for multi-dimensional recursion using both methods. Let us remark that the construction schema of [9] is obtained from Lemma 22, starting initially with the Petersen graph, using $\mathcal{P}_{10}^{*}(u, v)$ for edge replacement, two isolated vertices (see Figure 2, center) as the only expansion graph, and recursively applying the same technique to the obtained graphs. 


\subsection{Various g-edges with the same 5-capacity}

Quite obviously:

Proposition 26. Given an integer $k \geq 2$ and a graph $G, \phi_{c}(G)<k$ if and only if $0 \in C P_{k}\left(G_{u, v}\right)$ for a pair (equivalently all pairs) of vertices $u$ and $v$ of $G$.

Consequently, any $A$-edge where $0 \notin A$ is a graph in $F_{\geq 5}$, and, if it has the right girth and connectivity, it can be turned into a snark in $S_{\geq 5}$, via vertex expansion. In our list of sixteen sets from $G I_{5}$, there are ten that do not include 0 . Of the smaller g-edges representing each capacity (13 are depicted in Figure 1), the only one that meets the required cyclically 4-edge-connectivity is the Petersen graph, listed as a $(0,0)$-edge. Indeed $\mathcal{P}_{10} \in S_{\geq 5}$. It is cubic and no expansion is required here. However, for each $A \in G I_{5}$, there are many distinct $A$-edges of higher connectivity. One way to construct such g-edges is the replacement of one, or more, simple edges, by the 3-edge-connected thick $(1,4)$-edge. Let us show one detailed example: The Butterfly graph (see Figure 3) is a $(1,4)$-edge with terminals $u$ and $v$. It is, therefore, in $F_{\geq 5}$. The graph is cyclically 4-edge-connected and can be turned into a (actually many) snark $S \in S_{\geq 5}$, by expansion of vertices of high degrees. Here is how it is built: Starting with a thick $(1,4)$-edge, $G$ with terminals $u$ and $v$, the subgraph $Q$, circled on the right "wing" is a thick $(1,4)$-edge, which replaces a simple edge $x y$ of $G$. Similarly replaced is a simple edge $y t$ on the left wing. Replacements of simple edges by any $(1,4)$-edges do not affect the existence of sub-5-MCNZF's in a graph.

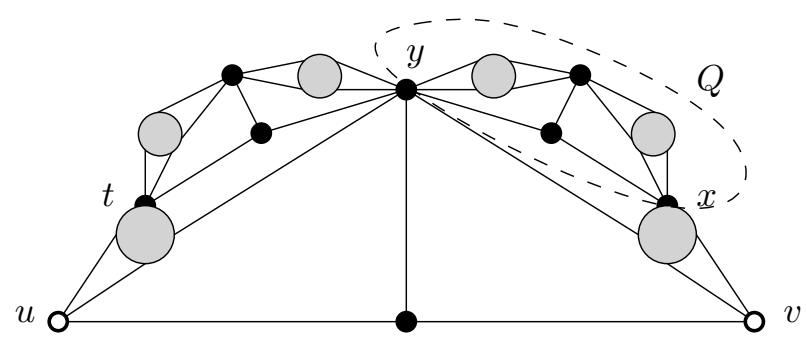

Figure 3: The Butterfly $(1,4)$-edge.

Similarly, an unlimited set of alternative g-edges can be constructed to represent each capacity $A \in G I_{5}$, from which snarks $S \in S_{\geq 5}$ can be constructed, of all shapes and sizes. 
Yet another simple observation: If an $A$-edge in a graph $G$ is replaced by a $B$-edge with $B \subseteq A$, it does not give rise to any sub-5-MCNZF in the obtained graph $G^{\prime}$, which does not exist in $G$. In particular, if $G \in F_{\geq 5}$ so is $G^{\prime}$. It is easy to verify that, if an edge $u v$ is removed from a graph $G \in F_{\geq 5}$, then the capacity of the obtained g-edge $(G-u v)_{u, v}$ is either $(4,1)$, or $\emptyset$. Considering the observation above, any such g-edge can replace any $(4,1)$-edge to generate new members of $F_{\geq 5}$, from previously generated ones.

One almost last peek into that seemingly vast bag of tricks: To push any cubic graph (containing only simple edges) into $F_{\geq 5}$, in a single step, just replace two adjacent (simple) edges by a pair of $(2,3)$ edges. The flow on the third edge incident with their common endvertex is now restricted to $(2,3)+(2,3)=(4,1)$. Yet, as that simple third edge is of capacity $(1,4)$, no sub-5-CNZF exists in the obtained graph, as promised. If aiming toward snarks, the $(1,4)$ component of each of the $(2,3)$-edges, should be thick, to provide the required edge-connectivity.

We kept the simplest trick to the very end of the list: Insert a single $\emptyset$-edge anywhere (make it thick to allow snarks). No sub-5-flow is admitted anymore.

\subsubsection{Redundancy}

The construction methods described so far are by no means independent. In fact, overlapping is rather wide and the same graphs and snarks are generated in many ways. Here are a few of many examples:

Let $(G-u v)_{u, v}$ be a g-edge, obtained by the removal of an edge $u v$ from $G \in F_{\geq 5}$, as described in Section 5.3. If $u v$ belongs to an expansion subgraph $H$, used as part of the construction of $G$, then any use of $(G-u v)_{u, v}$ as an edge, to construct a new graph is equivalent to replacing $H$ by a larger expansion graph $H^{\prime}$, when constructing $G$. Since the selection of an expansion graph is arbitrary, $H^{\prime}$ could have been selected to start with. So using $(G-u v)_{u, v}$ as an edge replacement is entirely redundant here.

Similarly, let $G^{\prime}$ be obtained from a graph $G$ by means of Corollary 19 . When applying Lemma 22 to $G^{\prime}$, if the cycle $C$ is contained in $G$, then the entire new construction can be considered as part of a larger initial graph, selected instead of $G$. Using Lemma 22 in that case is, therefore, redundant.

When elaborating on the usage of Lemma 22 in Section 5.2, we did count $T=\{(2,3)\}$ among the relevant subsets of $G I_{5}$. However, as specified on the penultimate paragraph of Section 5.3 , two consecutive $(2,3)$-edges turn any 
initial graph into $F_{\geq 5}$. Nothing is gained by starting from a graph already in $F_{\geq 5}$ and replacing an entire cycle.

When carefully checking the details, the last trick of Section 5.3 (in its "thick" version) is equivalent to the selection of an arbitrary graph, for expansion of a vertex of degree 5, in the graph depicted in Figure 2, left.

Despite these (and many other) causes of redundancy, the part of $S_{\geq 5}$ established by methods which were developed along this article, appears to be pretty rich. Significant support to that claim is provided in the next section.

\section{NP-Completeness}

Less than a decade ago, the only known snark with circular flow number 5 was the Petersen graph. An infinite family of such snarks was presented in [9], yet, as we demonstrated in previous sections, the entire collection $S_{\geq 5}$ of snarks with flow number 5 (or more?) is much richer than that. We now utilize the power of our construction methods to show that $S_{\geq 5}$ is, in fact, rich and complex enough, to facilitate an NP-completeness proof. In other words, that set of snarks is rich and dense enough, so that every instance $I$ of any Co-NP problem, can be emulated, by a snark $S \in S_{\geq 5}$, whose size and complexity are of polynomial order, in comparison with the size of $I$.

Theorem 27. Given an input snark $G$, deciding if $\phi_{c}(G)<5$ is NP-Complete.

We first prove a somewhat weaker version, where no restrictions, related to connectivity, girth, or vertex degrees are imposed on the input graph:

Lemma 28. Given any input graph $G$, deciding if $\phi_{c}(G)<5$ is NP-Complete.

Proof. A 3-hypergraph $H$ consists of a finite set $X$ of nodes and a collection of three element subsets of $X$, called triplets. The following problem is known to be NP-complete: Given a 3-hypergraph $H$, can the set of nodes be partitioned into two "color" sets, so that no triplet contains three nodes of the same color. If such a partition exists, then $H$ is said to be 2-colorable.

Given a 3-hypergraph $H$, we show that a graph $G(H)$ can be constructed, in polynomial time, such that $\phi_{c}(G(H))<5$ if and only if $H$ is 2-colorable.

Constructing $G(H)$. Each node $x$ is represented by a node-cycle $C(x)$. The length of $C(x)$ is twice the number of triplets that contain $x$. The 
edges of $C(x)$ are all of capacity $(1,2) \cup(3,4)$. The vertices along $C(x)$ are alternately referred to as positive and negative terminals of $C(x)$.

Each triplet $T$ is represented by a triplet-cycle $C(T)$, which consists of six simple $(1,4)$-edges, and six vertices $\left(T_{1}^{+}, T_{2}^{+}, T_{3}^{+}, T_{3}^{-}, T_{2}^{-}, T_{1}^{-}\right)$in that (cyclic) order.

Every occurrence of a node $x$ in a triplet $T$ is represented by two connector edges of capacity $(1,2) \cup(3,4)$, one between a positive terminal of $C(x)$ and a vertex $T_{i}^{+}$of $C(T)$, and the other one between a negative terminal of $C(x)$ and the vertex $T_{i}^{-}$(same index $i$ ) of $C(T)$. Each vertex of every node-cycle and every triplet-cycle is the endvertex of exactly one connector.

We choose, for reference, an orientation of $H$ where all node-cycles and triplet-cycles are directed cyclically, and the connectors are directed from node-cycles toward triplet-cycles.

We split the main statement into two separate propositions.

Proposition 29. If $H$ is 2-colorable then $\phi_{c}(G(H))<5$.

Proof. Let $\left(X_{1}, X_{2}\right)$ be a partition of $X$ which yields a 2-coloring of $H$, that is, every triplet $T$ includes one node from one set of the partition and two nodes from the other set.

To define a sub-5-MCNZF $f$ in $G(H)$ : We select a positive number $0<\epsilon<$ $1 / 6$ and assign $f$ values to the edges along every node-cycle, alternately $2-\epsilon$ and $-(2-\epsilon)$. Consequently, the $f$ values of the connectors incident with the terminals of each node-cycle are alternately $t$ and $-t$, where $t=1+2 \epsilon$.

If $x \in X_{1}$, then we set $f(p)=t$, for every connector edge $p$ incident with a positive terminal of $C(x)$, and $f(n)=-t$ for every connector $n$ incident with a negative terminal.

Conversely, if $x \in X_{2}$, then $f(p)=-t$, for every connector edge $p$ incident with a positive terminal of $C(x)$, and $f(n)=t$ for every connector $n$ incident with a negative terminal. Observe that $\pm(2-\epsilon)$ and $\pm(1+2 \epsilon)$ indeed belong to the capacity $(1,2) \cup(3,4)$.

It remains to define $f$ on the edges of the triplet cycles. Consider a triplet $T$. Since $\left(X_{1}, X_{2}\right)$ yields a 2 -coloring, the flow $f$ equals $t$ on one of the three connectors incident with $T_{1}^{+}, T_{2}^{+}, T_{3}^{+}$, and $f$ equals $-t$ on the other two, or vice versa. Either way, the (cyclic) sequence of flow values on the six connectors incident with vertices of $C(T)$ is either $(t,-t, t,-t, t,-t)$, or $(t, t,-t, t,-t,-t)$, or obtained from the second by reversing the order (Note 
that, as the sequence is cyclic, it does not necessarily start at $T_{1}^{+}$, but at any conveniently selected "first" vertex).

We now assign $f$ value $t$ to the "closing" edge of $C(T)$, that is, the edge going from the "sixth" vertex to the "first" one on the sequence, as listed above. The $f$ values of the edges along $C(T)$ then become $(t, 2 t, t, 2 t, t, 2 t)$, or $(t, 2 t, 3 t, 2 t, 3 t, 2 t)$. Recall that $t=1+2 \epsilon$ with $0<\epsilon<1 / 6$. Consequently, $t, 2 t$ and $3 t$ are all valid flow values in $(1,4)$. That remains true also if the order is reversed and the obtained values become $-t,-2 t$ and $-3 t$. For every edge $e$ of $G(H)$, the flow $f$ satisfies $f(e) \in C P_{5}(e)$, so it is indeed a sub-5-MCNZF in $G(H)$.

Proposition 30. If $\phi_{c}(G(H))<5$ then $H$ is 2-colorable.

Proof. Let $f$ be a sub-5-MCNZF in $G(H)$. By Lemma 18, the flow values on the edges along each node-cycle $C(x)$ alternately belong to $(1,2)$ and to $(3,4)$. Consequently, the values on the connectors incident with terminals of $C(x)$ alternately belong to $(1,3)$ and to $(2,4)$. As the capacity of a connector is $(1,2) \cup(3,4)$, the actual values alternate between $(1,2)$ and $(3,4)$. Let $X_{1}$ be the set of nodes $x$, for which $f(p) \in(1,2)$ on the connectors $p$, incident with positive terminals of $C(x)$, and let $X_{2}$ be the set of nodes $x$, for which $f(p) \in(3,4)$ on the connectors $p$, incident with positive terminals of $C(x)$. We claim that $\left(X_{1}, X_{2}\right)$ yields a 2-coloring of $H$. Assume, to the contrary, that this is not the case, then there exists a triplet $T$ such that the three $f$ values $d_{1}, d_{2}, d_{3}$ on the connectors incident with $T_{1}^{+}, T_{2}^{+}, T_{3}^{+}$, all belong to the same unit interval, say, to $(1,2)$. In that case, at least one of the four $f$ values, $a, a+d_{1}, a+d_{1}+d_{2}, a+d_{1}+d_{2}+d_{3}$ (on the four consecutive edges of $C(T)$, starting with $f\left(T_{3}^{-} T_{1}^{+}\right)=a$ ), belongs to $(4,1)$, in contradiction to $f$ being a sub-5-MCNZF. The same contradiction holds if $d_{1}, d_{2}$ and $d_{3}$ belong to $(3,4)$.

Propositions 29 and 30 are combined to yield Lemma 28.

We are now set to prove the stronger Theorem 27.

Proof. Theorem 27 is proved by converting the graph $G(H)$, described in the proof of Lemma 28, in polynomial time, into a snark $S(H)$, such that $\phi_{c}(S(H))<5$ if and only if $\phi_{c}(G(H))<5$.

As a snark, $S(H)$ should be of the right girth and connectivity. We should be, therefore, more specific about the structure of $G(H)$. Recall that a $(1,2) \cup(3,4)$-edge is a serial join of a $(3,2)$-edge and a $(1,4)$-edge. We 
use the minimal $(3,2)$-edge and the thick $(1,4)$-edge (see Figure1, top right) for the edges along each node-cycle. For connectors, on the other hand, we use a simple edge as the $(1,4)$ component. Also, of the two terminals of a connector, the one which belongs to the simple edge is selected to be attached to the triplet-cycle. That way, every vertex of every triplet-cycle is incident with three simple edges and it is of genuine (not "generalized") degree 3. A part of a node cycle and its incident connectors is depicted in Figure 4. It is easily verified that $G(H)$ is now cyclically 4-edge-connected and of girth at least 5. As a snark, the circular flow number of $S(H)$ should be larger than 4. To guarantee that, we first prove:

Proposition 31. Regardless of the 3-hypergraph $H$, being 2-colorable or not, $\phi_{c}(G(H))>4$.

Proof. Recall the definition of $\mathcal{P}_{10}^{*}(u, v)$, as the g-edge obtained from the Petersen graph $\mathcal{P}_{10}$, by the removal of an edge $u v$. The range of a 4-MCNZF is the closed interval $[1,3]$ modulo 4 . The Petersen graph $\mathcal{P}_{10}$ does not admit a 4 -MCNZF. As a result, the set of modulo 4 flow values that can be "pushed" through $\mathcal{P}_{10}^{*}(u, v)$ with the flow on each edge restricted to $[1,3]$ is disjoint from $[1,3]$. Since that set is a union of closed unit intervals of $\mathbb{R} / 4 \mathbb{Z}$ (following the same argument that leads to Lemma 14), it consists solely of the point 0 . The same holds for the parallel join of two such g-edges, which forms the $(3,2)$ component of each connector of $G(H)$. As a result, a modular-4-flow on the simple edge component of a connector is also restricted to 0 , so no 4-MCNZF is possible.

It remains to take care of vertices $v$ with degree $d(v)>3$. That is achieved, by an expansion of each such vertex $v$, into a cubic subgraph. By Proposition 24, an expansion of a vertex never decreases the circular flow number. The tricky part is to guarantee $\phi_{c}(S(H))<5$ whenever $\phi_{c}(G(H))<$ 5. Luckily, the structure of each node-cycle is highly symmetric and it is basically the same for every node-cycle and every graph $G(H)$. Figure 4 illustrates the basic component of which $G(H)$ is made - $\mathrm{A}(1,2) \cup(3,4)$ edge of a node-cycle and the incident connector. We refer to that subgraph as a link. All links in all graphs $G(H)$ are isomorphic. A link includes four vertices of degree larger than 3, labeled on Figure 4 as $x$ of degree 11, $y$ of degree $7, z$ of degree 5 and $w$ of degree 5 . Vertices $x, y, z$, and $w$ will be respectively replaced by four expansion graphs $X, Y, Z$ and $W$. 


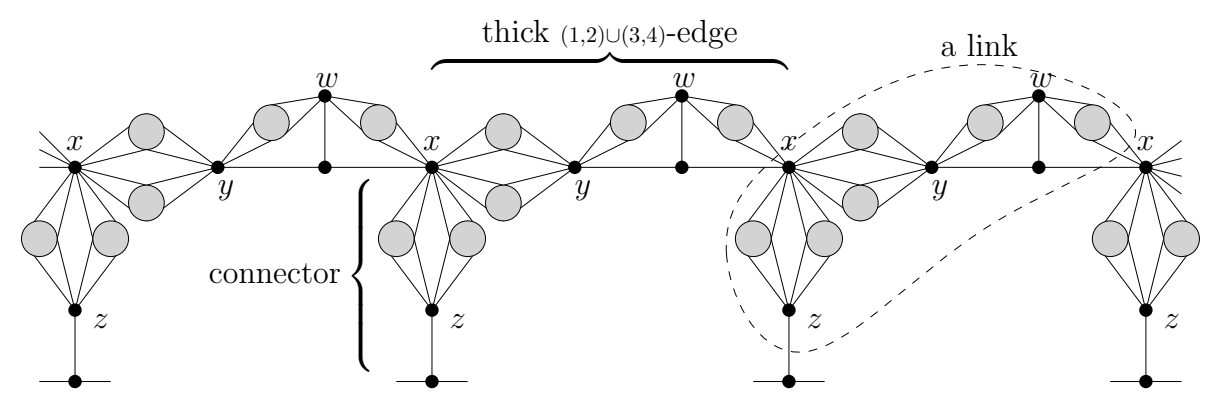

Figure 4: A segment of a node-cycle with incident connectors

Consider the sub-5-MCNZF $f$ defined in the proof of Proposition 29. After selecting $0<\epsilon<1 / 6$, say, $\epsilon=1 / 12, f$ is fully defined, up to switching the sign of all $f$-values on a node cycle and the incident connectors (by switching the roles of its positive and negative terminals). The flow $f$ is then defined for every edge of a link, and it is the same (up to reversing all signs) for all links. The expansion graphs $X, Y, Z$ and $W$ should be designed to allow a sub-5-MCNZF, which maintains the $f$ values on all original edges of the link. An expansion graph suitable for that task, also clearly fits if all flow values on the incident edges switch their signs. Consequently, all we need are four fixed expansion graphs $X, Y, Z$ and $W$, to be used for all links and convert every graph $G(H)$ into a snark $S(H)$, as required, in linear time.

Explicit construction and presentation of these four graphs are certainly doable. However, that somewhat tedious mission, can be spared here. It suffices to show that such expansion graphs do exist, and that claim was already proved, in a more general setting.

In [8], Sections 3 and 4, the authors present a procedure to convert a cyclically 4-edge-connected graph $G$, of girth at least 5 and such that $4<$ $\phi_{c}(G)<5$, into a snark $S$ with the same circular flow number. Starting with a given $r$-CNZF $f$ in $G$, they remove each vertex $v$ of degree $d(v)>3$ and reroute the edges incident with that vertex, through a certain "network", which makes the obtained graph $S$ a snark. The structure of each such network is derived from the values of $f$ on the edges incident with $v$. A new $r$-CNZF is defined in $S$, whose restriction to the original edges of $G$ is the given flow $f$. The following lemma summarizes that part of [8], although it is not explicit in that article:

Lemma 32. Let $g$ be an $r$-CNZF in a cyclically 4-edge-connected graph $G$, of 
girth at least 5 and such that $4<\phi_{c}(G)<5$. By means of expansion of each vertex of degree larger than 3 (possibly accompanied by smoothing of vertices of degree 2), a snark $S$ is obtained from $G$. There exists an $r$-CNZF $g_{1}$ in $S$, such that $g_{1}(e)=g(e)$ for every edge $e \in E(G)$.

Lemma 32 relates to a (real valued) $r$-CNZF. For our purpose, it should be reformulated in terms of modular flows.

Let $f$ be an $r$-MCNZF in (a reference orientation $D_{0}$ of) $G$. By Proposition 10, there exists an $r$-CNZF $g$, on an orientation $D$ of $G$, such that, for every edge $e$ of $G, g(e) \equiv f_{D}(e)$ modulo $r$.

If $G$ is cyclically 4 -edge-connected, of girth at least 5 and such that $4<$ $\phi_{c}(G)<5$, then Lemma 32 asserts the existence of expansion graphs, one for every vertex $v$ of degree $d(v)>3$, which turn $G$ into a snark $S$ with an $r$-CNZF $g_{1}$, whose restriction to $E(G)$ equals $g$. Let $\tilde{f}=\left(g_{1}\right.$ modulo $\left.r\right)$. That is, $\tilde{f}$ is an $r$-MCNZF in $S$, obtained from $g_{1}$ by interpreting each $g_{1}(e)$ as a residue in $\mathbb{R} / r \mathbb{Z}$. The restriction of $\tilde{f}$ to $G$ is clearly $f_{D}$. The orientation does not really matter. For simplicity's sake we can switch back to the reference orientation $D_{0}$ and the flow $f$.

In summary:

Lemma 33. Let $f$ be an $r$-MCNZF in a cyclically 4-edge-connected graph $G$, of girth at least 5 and such that $4<\phi_{c}(G)<5$. By means of expansion of each vertex of degree larger than 3 (possibly accompanied by smoothing of vertices of degree 2 ), a snark $S$ is obtained from $G$. There exists an $r$-MCNZF $\tilde{f}$ in $S$, such that $\tilde{f}(e)=f(e)$ for every edge $e \in E(G)$.

Clearly, the structure of each expansion graph for a vertex $v$ depends solely on the values of $f$ on the edges incident with $v$. The existence of the expansion graphs $X, Y, Z$ and $W$ required for our construction, immediately follows.

Let us reemphasize that the converting procedure of [8] is not a part of our reduction algorithm and its details and complexity are irrelevant. It is used here only as an existence assertion, to save an explicit presentation of the four expansion graphs.

$S(H)$ is constructed by replacing each vertex $x, y, z$ and $w$, in every link of $G(H)$, by expansion graphs, $X, Y, Z$ and $W$, all in linear time. 


\subsection{Analogous results for $r \in(4,5)$}

As previously stated, using open capacities seems to be a must when dealing with sub-5-flows. However, it may also come in handy when smaller values of $r$ are considered.

The Petersen graph $\mathcal{P}_{10}$ is known to admit an (integer) 5-NZF $f$, such that $f(u v)=4$ for exactly one edge $u v$, and $f(e) \in\{1,2,3\}$ for all other edges $e$. That, combined with $\phi_{c}\left(\mathcal{P}_{10}\right)=5, \phi_{c}\left(\mathcal{P}_{10}^{*}\right)=4$ and Proposition 10 , lead to the following (which is also stated in [11] for the closed capacity):

For every rational $r \in(4,5)$ the closed $r$-capacity of $\mathcal{P}_{10}^{*}(u, v)$ is $[4, r-4]$ (modulo $r$ ) and the open $r$-capacity of the same g-edge is the open interval $(4, r-4)$ with the same boundaries. Lemma 3.5 of [11] states the existence of a collection of g-edges, among them one with closed $r$-capacity $[r-1,1]$. Let this g-edge be denoted here by $Q_{r}$. Following the proof of Lemma 3.5 in [1], $Q_{r}$ is constructed by repeatedly applying serial and parallel joins, starting with copies of $\mathcal{P}_{10}^{*}(u, v)$ and simple edges (of closed $r$-capacity $[1, r-1]$ and open $r$-capacity $(1, r-1))$.

In general, open and closed capacities cannot be switched carelessly. See the discussion following Definition 13. However, the serial-parallel technique, as well as the set sum and intersection operations, result in analogous outcomes when applied to open and closed intervals with the same boundaries. Accordingly, the open $r$-capacity of $Q_{r}$ is $(r-1,1)$.

Replacing the g-edge $\mathcal{P}_{10}^{*}$ in our constructions, by $Q_{r}$, allows the generalization of many of our results, from $r=5$, to $r \in(4,5]$. In particular, meaningful results can be obtained when using (even if implicitly) the analogues of Lemma 18 and Corollaries 20 and 19 , which strongly rely on the involved capacities being open capacities.

For example

Theorem 34. For every rational $r \in(4,5)$, deciding whether $\phi_{c}(S)<r$, for an input snark $S$ is NP-Complete.

Proof. (outlines) The proof accurately follows those of Lemma 28 and Theorem 27, subject to the following adaptations:

- $Q_{r}$ replaces every $\mathcal{P}_{10}^{*}(u, v)$ (the o 0 so shape in Figures 1 and 4 ) of $G(H)$. Accordingly:

- Edges of the node-cycles and the connectors are of $r$-capacity $(1,2) \cup$ $(r-2, r-1)$ 
- The $(1, r-1)$ component of a connector is a thick $(1, r-1)$-edge (see Figure 1, top right).

- The parameter $\epsilon$ selected for the definition of $f$ in the proof of Proposition 29, should satisfy $0<\epsilon<(r-4) / 6$.

- In addition to the four vertices $x, y, z$ and $w$, there are other vertices $v$ of degree $d(v)>3$ within every $Q_{r}$ subgraph. However, the set of such vertices in a link is still finite and so is the set of required expansion graphs. With a finite fixed set of expansion graphs, the construction of $S(H)$ from $G(H)$ is still performed in linear time.

- Every detail of the proofs of Lemma 28 and Theorem 27, straightforwardly translates into this modified setting.

\section{Concluding remarks, open problems and directions for further research}

\subsection{How actually rich is $S_{\geq 5}$ ?}

As diverse as they may seem, snarks constructed in this article still share certain restricting characteristics. They are all based on the $(4,1)$-edge $\mathcal{P}_{10}^{*}(u, v)$, obtained from the Petersen graph, and, as such, they all contain $\mathcal{P}_{10}^{*}(u, v)$ subgraphs, and they all (other than $\mathcal{P}_{10}$ ) are no more than cyclically 4-edgeconnected.

High edge-connectivity is known to be correlated with smaller flow number, so the above may hold for every $S \in S_{\geq 5}$, or even every graph $G \in F_{\geq 5}$. We hesitate to call it a "conjecture" (to replace Mohar's Strong 5-flow Conjecture [10]). It is, however, definitely an intriguing question, which we are not the first to post (e.g. [8]):

Problem 35. Is there a cyclically 5-edge-connected graph $G \in F_{\geq 5}$, other than $\mathcal{P}_{10}$ ?

Or at least: 
Problem 36. Is there a graph $G \in F_{\geq 5}$ with no $\mathcal{P}_{10}^{*}(u, v)$ induced subgraph, possibly with each of the two terminals $u$ and $v$ split into two pairs $\left(u_{1}, u_{2}\right)$ and $\left(v_{1}, v_{2}\right)$, due to vertex expansion? Here $\mathcal{P}_{10}$, the parallel join of $\mathcal{P}_{10}^{*}(u, v)$ and a simple edge is no exception.

Positive answers to either one of the above, or to both, would mean that the borders of $F_{\geq 5}$ and $S_{\geq 5}$ are far beyond those drawn by our methods.

Assuming that this is not the case, then every $G \in F_{\geq 5}$ is only cyclically 4-edge-connected and contains a $\mathcal{P}_{10}^{*}(u, v)$ subgraph. Does this mean that the construction tools developed in Sections 4 and 5 can generate every member of $F_{\geq 5}$ and $S_{\geq 5}$ ?

This last question is not entirely well defined. We did not present a systematic list of construction methods, but only sporadically demonstrated some. It might be beneficial to try and make such a list, and then to study how extensively the various methods overlap. Is it possible to define, or to get close to a "basis" of independent (or almost independent) constructing operations, which can be combined to produce all other constructing methods?

We have, however, systematically analyzed the set of graphic 5-capacities $G I_{5}$. Here a well defined question is in place:

Problem 37. Does the list of 5-capacities, presented in Section 4, include all members of $G I_{5}$ ?

A similar question can be asked with regards to $G I_{3}$ (and $G I_{4}$, though we suggested no list for that one).

\subsection{3-poles - The two dimensional case}

Similar to a $g$-edge, a 3-pole is a "network" $H$ with three terminals - a source $u$ and two sinks $v$ and $w$. As part of a graph $G$, a 3-pole $H$ is a subgraph, which shares its three terminals, and is otherwise vertex-disjoint, from the rest of the graph. The (open or closed) capacity of a 3-pole is the set of pairs $(x, y)$ of flow values, which can be simultaneously pushed from $u$ to $(v, w)$ : That is, $x$ from $u$ to $v$ and $y$ from $u$ to $w$, subject to given restrictions on the flow values on edges of $H$. The open $k$-capacity of a 3 -pole, would then be, as a generalization of Definition 13 , a set of points $(x, y)$ on the torus $(\mathbb{R} / k \mathbb{Z})^{2}$, obtained that way, where the flow values on the edges of $H$ are restricted to $(1, k-1)$ modulo $k$. Similarly to Lemma 14 , the open $k$-capacity of a 3 -pole 
is a symmetric (with respect to the origin point $(0,0)$ ) union of open convex integer polygons on $(\mathbb{R} / k \mathbb{Z})^{2}$. An integer polygon is a polygon whose vertices have two integer coordinates. Two 3-poles which share a common source $u$ and two distinct pairs of sinks, $\left(v_{1}, w_{1}\right)$ and $\left(v_{2}, w_{2}\right)$ with $k$ capacities $A$ and $B$, can be merged to form parallel and serial joins. The capacity of a parallel join is $A+B$. The capacity of a serial join, however, is not the intersection but the composition $A \circ\{(-y, z) \mid(y, z) \in B\}$ of $A$ and $\{(-y, z) \mid(y, z) \in B\}$ as binary relations in $\mathbb{R} / k \mathbb{Z}$. Neither the parallel join, nor the serial is uniquely defined. We leave the details for further research. Anyway, there are finitely many potential $k$-capacites to generate from $k^{2}$ integer points in $(\mathbb{R} / k \mathbb{Z})^{2}$. Definitely, if $k$ is small, the mission is within the reach of a computer assisted comprehensive study.

\subsection{Other applications to Nowhere-Zero flow problems}

Closed capacities of g-edges were very successfully applied in [11] to the study of $r$-CNZF where $r<5$. However, the authors only considered the serial-parallel mechanism and there was no attempt to characterize the set of all these capacities, similar to what we did for the set $G I_{5}$ of all open 5-capacities. Systematic development of the subject may lead to further applications in the study of Nowhere-Zero Flows. The same holds for 3-poles and multi-poles of higher dimension with respect to any $r \in(4,5]$ with closed, as well as open capacities.

\subsection{Regular matroids and graph coloring}

Lemma 14 can be stated and proved in the wider setting of totally unimodular integer programming. As such, it can be applied to flows in general regular matroids, rather than just graphs. Particularly interesting may be the cographic case, where the analogue of an $r$-CNZF is a tension function, induced by a proper $r$-circular coloring of a graph. $k$-Co-capacities, may appear to be useful for the study of circular graph coloring (where $r$ is not bounded by 5 or 6 or any other upper bound). 


\section{References}

[1] A. Bondy and P. Hell, A note on the star chromatic number, J. Graph Theory 14(4) (1990), 479-482.

[2] G. Brinkmann, Catalogue of snarks of the orders 10-30, electronic document, Universität Bielefeld, Bielefeld, 1998.

[3] L. Esperet, M. Montassier, P. Ochem, and A. Pinlou, A complexity dichotomy for the coloring of sparse graphs, J. Graph Theory 73(1) (2013), 85-102.

[4] L.A. Goddyn, M. Tarsi, and C.Q. Zhang, On $(k, d)$-colorings and fractional nowhere-zero flows, J. Graph Theory 28(3) (1998), 155-161.

[5] J. Hägglund, On snarks that are far from being 3-edge colorable, Manuscript, 2012. arXiv:1203.2015.

[6] F. Jaeger, Nowhere-zero flow problems, in: L. Beineke, et al. (Eds.), Selected Topics in Graph Theory, vol. 3, Academic Press, London, New York, 1988, pp. 91-95.

[7] M. Kochol, Hypothetical Complexity of the Nowhere-Zero 5-Flow Problem, J. Graph Theory 28 (1998), 1-11.

[8] R. Lukot'ka and M. Skoviera, Snarks with given real flow numbers, J. Graph Theory 68(3) (2011), 189-201.

[9] E. Máčajová and A. Raspaud, On the Strong Circular 5-Flow Conjecture, J. Graph Theory 52(4) (2006), 267-357.

[10] B. Mohar, Problem of the Month, http://www.fmf.uni-lj.si/ mohar/Problems/P03034CircularFlowConjecture.html, March and April 2003.

[11] Z. Pan and X. Zhu, Construction of Graphs with Given Circular Flow Numbers, J. Graph Theory 43(4) (2003), 304-318.

[12] E. Steffen, Circular flow numbers of regular multigraphs, J. Graph Theory 36 (2001), 24-34. 
[13] W.T. Tutte, A contribution to the theory of chromatic polynomials, Canad. J. Math. 6 (1954), 80-91.

[14] W.T. Tutte, On the algebraic theory of graph colorings, J. Combin. Theory 1 (1966), 15-50.

[15] C.Q. Zhang, Integer Flows and Cycle Covers of Graphs, New York: Marcel Dekker 1997. 